Case Report

Open Access

\title{
Specimen Exteriorization in Laparoscopic Colon Surgery: A Handy Alternative to the Gel Port
}

\author{
Bethany Malone ${ }^{\star}$ Erica Laipply and Frederick Slezak \\ Department of Colorectal Surgery, Summa Health System, Akron, OH, USA
}

\section{Article Info}

*Corresponding author:
Bethany Malone
Department of Colorectal Surgery
Summa Health System
95 Arch Street, Suite 150
Akron, OH 44304
USA
E-mail: maloneb@summahealth.org

Received: May 19, 2018

Accepted: June 7, 2018

Published: June 12, 2018

Citation: Malone B, Laipply E, Slezak F. Specimen Exteriorization in Laparoscopic Colon Surgery: A Handy Alternative to the Gel Port. Madridge J Surg. 2018; 1(2): 38-39. doi: $10.18689 / \mathrm{mjs}-1000109$

\section{Copyright: (c) 2018 The Author(s). This work is licensed under a Creative Commons Attribution 4.0 International License, which permits unrestricted use, distribution, and reproduction in any medium, provided the original work is properly cited.}

Published by Madridge Publishers

\begin{abstract}
Laparoscopic surgery has become the standard of care for many operations, including colectomies for various etiologies. Despite the benefits of these smaller incisions, a larger incision must still be made to deliver the specimen. This incision releases the insufflation necessary to maintain laparoscopic visualization and working space. Using standard operating room equipment consisting of a sterile glove, wound protector, and silk ties, the authors propose a novel technique for restoring and maintaining insufflation after specimen delivery. This technique is more cost effective and universally accessible when compared to specialized port systems.
\end{abstract}

Keywords: Specimen exteriorization; Laparoscopic colon surgery; Gel port.

\section{Introduction}

When compared to classic open surgery, laparoscopic colon and rectal surgery has been shown to have decreased lengths of stay, earlier return of bowel function, and equivalent oncologic outcomes [1]. However, no such differences have been shown between purely laparoscopic or laparoscopic assisted surgical techniques [2]. Whichever minimally technique is selected, an incision must be made to deliver the specimen. When the procedure is performed purely laparoscopically, a wound retractor is placed over the incision to prevent seeding of the specimen delivery site [3]. In the laparoscopic or hand assisted method, a gel port is used which is significantly more expensive than a wound retractor. The gel port allows delivery of the specimen while maintaining insufflation so that the port can continue to be used for the introduction and utilization of laparoscopic equipment. We propose a novel method that combines the flexibility of a hand assisted gel port with the thrift of a wound retractor used in purely laparoscopic cases.

\section{Method}

The distal margin of the specimen is mobilized in standard laparoscopic fashion. After adequately mobilization, the infraumbilical port site is then extended to approximately $4 \mathrm{~cm}$ and a small Alexis wound retractor (Applied Medical, Rancho Santa Margarita, $C A$ ) is placed in the incision. The specimen is then exteriorized through this port site and the proximal division is performed extracorporeally. The specimen is then handed off the field and the remaining colon replaced into the patient. A Triumph latex glove size 5.5 (Medline Industries, Chicago, IL) is then secured over the Alexis port (Figure 1), and the tip of one of the fingers is trimmed with scissors. A $12 \mathrm{~mm}$ trocar is then placed through this trimmed finger and secured with a 3-0 silk tie (Figure 2). In sufflation is resumed and this trocar is available for the laparoscopic access necessary to complete the procedure (Figure 3 ). 


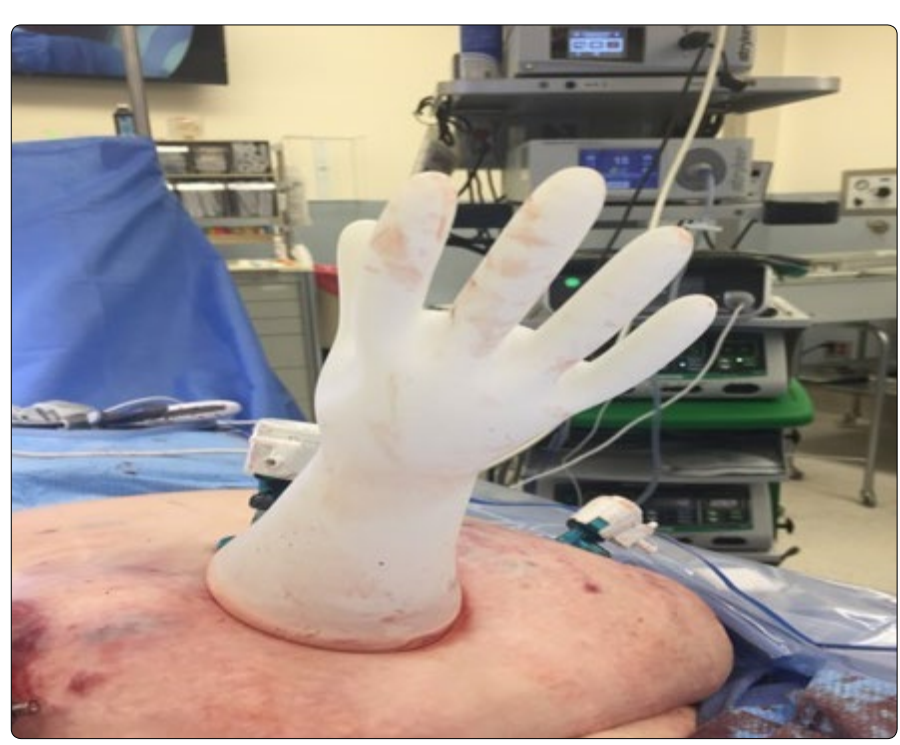

Figure 1. Glove secured over Alexis wound retractor

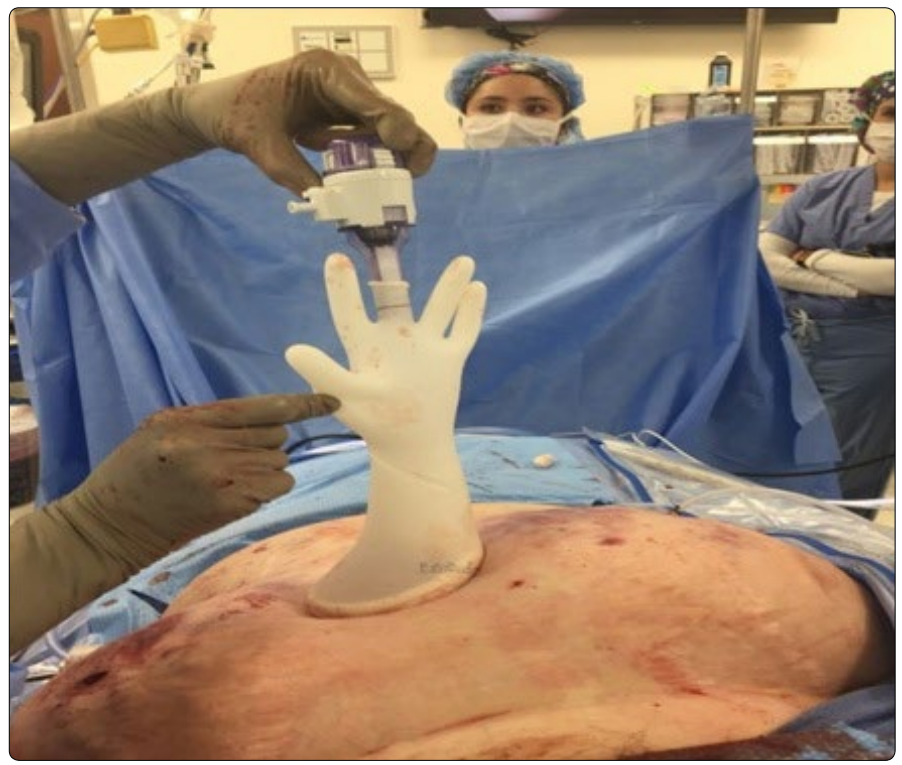

Figure 2. $12 \mathrm{~mm}$ trocar secured to glove with tie

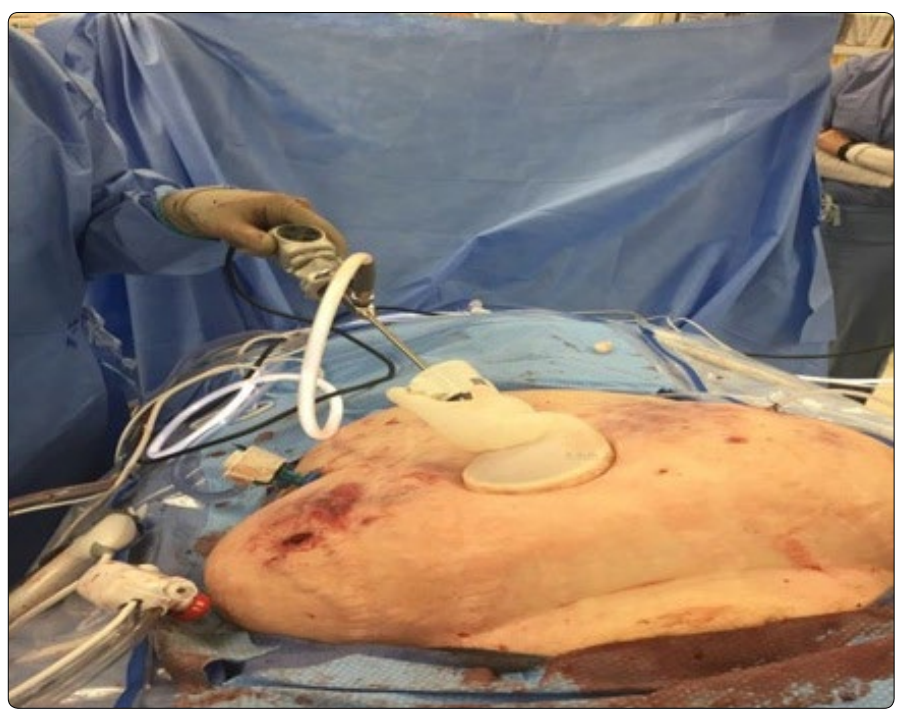

Figure 3. Trocar used to visualize remainder of case

\section{Discussion}

Laparoscopic surgery is steadily becoming the standard of care across the surgical specialties. While surgeons must remain adept at incorporating new technologies into clinical practice, we must also remember both cost containment and operating room efficiency. More specialized equipment such as gel ports may not be routinely stocked in all operating rooms. However, gloves of all materials and sizes are easily within the circulating nurse's reach. This minimizes trips out of the operating room for supplies and overall improves the efficiency of the entire operative theatre. Additionally, extra gloves are often open on the scrub tech's sterile field and can now be used judiciously to further the progression of the case.

\section{Conclusion}

We present both an economically and time conscientious alternative to hand assisted laparoscopic delivery of specimens in colorectal surgery utilizing standard operative equipment.

\section{Conflict of Interest}

The authors confirm that there is no conflict of interest regarding this manuscript.

\section{References}

1. Van der Pas MH, Haglind E, Cuesta MA, Furst A, Lacy AM, Hop WCJ, et al. Laparoscopic versus open surgery for rectal cancer (COLOR II): short-term outcomes of a randomized, phase 3 trial. Lancet Oncol. 2013; 14(3): 210218. doi: 10.1016/S1470-2045(13)70016-0

2. Koopmann $\mathrm{MC}$, Heise CP. Laparoscopic and minimally invasive resection of malignant colorectal disease. Surg Clin North Am. 2008; 88(5): 10471072. doi: 10.1016/j.suc.2008.05.009

3. Cirocco WC, Schwartzman A, Golub RW. Abdominal wall recurrence after laparoscopic colectomy for colon cancer. Surgery. 1994; 116(5): 842-846. 